Original Research

\title{
Adding a tibial tubercle osteotomy with anteromedialisation to medial patellofemoral ligament reconstruction does not impact patient-reported outcomes in the treatment of patellar instability
}

\author{
Danielle H. Markus, BA*, Eoghan T. Hurley, MB BCh MCh, Aaron Gipsman, MD, \\ Kirk A. Campbell, MD, Laith M. Jazrawi, MD, Michael J. Alaia, MD, Eric J. Strauss, MD \\ Department of Orthopedic Surgery, New York University Langone Health, 333 E $38^{\text {th }}$ Street, New York, NY 10016, USA
}

\section{A R T I C L E I N F O}

\section{Keywords:}

Knee

Joint instability

Ligaments

Sports medicine

Osteotomy

\begin{abstract}
A B S T R A C T
Objectives: An isolated medial patellofemoral ligament (MPFL) reconstruction (MPFLR) has been demonstrated to be an effective treatment option in the prevention of patellar instability, but there is growing support for performing a tibial tubercle osteotomy (TTO) in patients with an elevated tibial tubercle-trochlear groove distance. The purpose of this study was to evaluate the impact of adding a TTO to MPFLR on patient-reported outcomes. Methods: A retrospective review of patients who underwent MPFLR with or without TTO with a minimum of 12month follow-up was performed. Patients in both groups were matched based on age, gender, and follow-up time. Recurrent instability (including redislocation and subluxation), visual analogue scale (VAS) score, Kujala score, and satisfaction were evaluated.

Results: There were 59 patients who underwent MPFLR with concomitant TTO performed at our institution and met our inclusion and exclusion criteria. These patients were then matched to patients undergoing isolated MPFLR based on demographics and follow-up time. The mean age was 25.0 years, $76.3 \%$ were female, and the mean follow-up time was 49 months. There was a significant difference in mean tibial tubercle-trochlear groove distance (19.8 \pm 3.9 vs. $14.1 \pm 2.8)$ between groups. There was no significant difference in VAS (1.48 \pm 2.0 vs. $1.49 \pm 2.1, \mathrm{p}=0.972)$, satisfaction $(86.1 \% \pm 24.2 \%$ vs. $81.2 \% \pm 27.9, \mathrm{p}=0.311)$ or revision surgeries $(10.2 \%$ vs. $10.2 \%$ ) between groups.

Conclusion: Matched patients undergoing MPFLR with TTO compared with isolated MPFLR demonstrate no statistically significant difference in patient-reported outcomes, levels of pain, and satisfaction postoperatively. Furthermore, the addition of a TTO does not increase the risk of further surgery or complications.

Level of evidence: III, retrospective comparative study.
\end{abstract}

\section{What are new findings?}

- Matched patients undergoing medial patellofemoral ligament reconstruction (MPFLR) with anteromedialising tibial tubercle osteotomy (TTO) compared with isolated MPFLR demonstrate similar levels of pain and satisfaction postoperatively.

- Addition of a TTO does not increase the risk of further surgery or complications.

- Although the two groups have inherently different anatomic differences, this study proves that surgeons shouldn't fear the TTO!

\section{Introduction}

Lateral patellar instability is a common clinical pathology, comprising approximately $3 \%$ of all knee injuries and a leading cause of traumatic hemarthrosis seen in orthopaedic clinics [1]. The highest incidence is seen in patients aged 14-18, of which adolescent females are at particular risk [2-4]. A single patellar dislocation episode causing injury to the medial patellofemoral ligament complex may consequently result in recurrent instability, as the medial patellofemoral ligament (MPFL) is the primary stabilising soft tissue restraint to lateral patellar translation in early flexion. Dislocation events also may cause acute trauma to the joint such as articular cartilage lesions and osteochondral fractures $[5,6]$.

\footnotetext{
* Corresponding author. Department of Orthopedic Surgery, NYU Langone Health, 333 E $38^{\text {th }}$ St, New York, NY 10016, USA. Tel.: $+708-829-7668$.

E-mail address: Danielle.Markus@nyulangone.org (D.H. Markus).
} 
An isolated MPFL reconstruction (MPFLR) has been demonstrated to be an effective treatment option in the prevention of recurrent patellar instability episodes [7-10]. However, there is growing support for performing a bony realignment procedure such as a tibial tubercle osteotomy (TTO) in conjunction with MPFLR if an elevated tibial tubercle-trochlear groove (TT-TG) distance, patella alta, or excessive femoral anteversion is present $[11,12]$. The optimal course of management in the setting of patellar instability remains a challenge, as numerous surgical options exist without a clear consensus regarding superior surgical technique or graft choice [13,14]. Furthermore, although evidence exists supporting the roles trochlear morphology and malalignment play in patellar instability, concerns are present regarding the morbidity introduced with the addition of bony realignment procedures [15]. The literature surrounding whether the added benefit of a realignment procedure justifies the potential risk of complications is sparce and contradictory, as some authors posit that MPFLR can stand alone regardless of bony anatomy and that a TTO may not be required $[10,16]$.

The purpose of the present study was to evaluate the impact of adding a TTO with anteromedialisation to MPFLR on patient-reported outcomes and complications in the treatment of patellar instability. Our hypothesis was that the addition of a concomitant TTO would not result in differing patient-reported outcomes when compared with those receiving an isolated MPFL reconstruction.

\section{Methods}

\section{Patient selection}

This study received approval from our institutional review board. A retrospective review was carried out to identify all patients who underwent MPFLR between 2011 and 2019. The operative notes of all patients who underwent MPFLR with or without TTO for patellar instability were analysed. The inclusion criteria for this study were those undergoing MPFLR, skeletally mature, and a minimum follow-up of 12 months. Subsequently, patient matching between those undergoing MPFLR and TTO and isolated MPFLR based on patient demographics (age, gender, and follow-up length) was performed to generate two comparable groups.

\section{Surgical technique}

In those undergoing a TTO, a midline incision was made from the superior pole of the patella to two fingerbreadths distal to the tibial tubercle, approximately $8-10 \mathrm{~cm}$ in length. Using the femoral condyles and ankle as reference points, a guide pin was inserted into the tibial tuberosity perpendicular to the posterior tibia. The osteotomy cutting block was positioned using the guide pin onto the medial aspect of the tibial tuberosity. The cutting block was oriented in a posteromedial to anteromedial position and provisionally held in place with two pins. The proposed osteotomy cut was checked with the saw blade exit indicator. With the deep soft tissue retractor in place, the tibial tuberosity osteotomy was made using a sagittal saw at a 45-degree or 60-degree angle depending on the planned extent of tubercle anteromedialisation. The most distal aspect and the associated periosteum was left intact. The amount of anteromedialisation needed was determined based on imaging, TT-TG, and intraoperative stabilisation and was confirmed intraoperatively with a ruler. The tibial tuberosity was fixed using two $5.0 \mathrm{~mm}$ cortical screws (Arthrex, Naples, FL) inserted in lag by technique fashion, aiming slightly lateral to medial. The position and lengths of the screws were checked with intraoperative fluoroscopy.

MPFLR, either in isolation or following the TTO, was performed by inserting the two tails of a prepared gracilis allograft into tunnels created in the patella, one $4 \mathrm{~mm}$ from the superior pole, and the second $10 \mathrm{~mm}$ distal to the first. The allograft ends were fixed using two Swivel-lock suture anchors (Arthrex). Schottle's point was identified using intraoperative fluoroscopy, allowing a guide pin to be inserted. The graft was then tunnelled deep to the vastus medialis, and with tension held on the graft at the guide pin site, isometry was confirmed. A $6 \mathrm{~mm}$ reamer was then used over the guide pin, and the graft shuttled into the tunnel. The graft was tensioned with the patella reduced, and the knee in 60 degrees of flexion to avoid overconstraint and femoral sided fixation was performed using a $6 \times 23 \mathrm{~mm}$ biotenodesis screw (Arthrex).

\section{Rehabilitation protocol}

The pace of rehabilitation protocol differed based on whether or not a concomitant TTO was performed. In both groups, the knee was placed in a hinged brace locked in full extension postoperatively. In isolated MPFLR cases, weightbearing was advanced, and the hinged knee brace unlocked from 0 to 90 degrees of flexion at the initial postoperative visit, 6-10 days after surgery. Formal physical therapy was used working on knee range of motion and strengthening with a goal of 90 degrees of flexion to be achieved by postoperative week 6 at which point the hinged knee brace was completely unlocked. Patients were transitioned from the hinged brace to a patellar stabilising brace at postoperative week 12 . In combined TTO and MPFLR cases, progressive advancement of weightbearing and regaining of balance was started at postoperative week 6 , with radiographic evidence of early bony healing present. For both isolated and combined cases, return to contact in training was allowed after 4 months, while return to full contact and competition typically occurred at 6 months postoperatively. A return to full activities and sports without restrictions is dependent on each patient's individual progress, taking into consideration strength, range of motion, and pain.

\section{Data collection and clinical outcomes}

Data on patient characteristics and preoperative demographics were collected, including age, gender, laterality, and previous knee surgeries. Intraoperative and postoperative complications were recorded. Evaluation of postoperative patient-reported outcomes was carried out after telephone survey including visual analogue scale (VAS) score. Subjective Kujala score, satisfaction ("How satisfied are you with your procedure overall from 1-100?") and whether they would undergo the same surgery again were compared. Finally, recurrent instability events (including dislocations and subluxations) were recorded.

\section{Statistical analysis}

All statistical analysis was performed using GraphPad Prism 8.3 (GraphPad, La Jolla, CA). For all continuous and categorical variables, descriptive statistics were calculated. Continuous variables were reported as weighted mean and estimated standard deviation, whereas categorical variables were reported as frequencies with percentages. Categorical variables were analysed using Fisher's exact or chisquare test. The independent or paired $t$-test for normally distributed variables or the nonparametric Mann-Whitney $U$ test or Wilcoxon signed-rank test was performed to compare continuous variables. A value of $p<0.05$ was considered to be statistically significant.

\section{Results}

\section{Patient demographics}

Overall, there were 59 patients who underwent MPFLR with concomitant TTO performed at our institution that met our inclusion and exclusion criteria. A complete diagram outlining our inclusion and exclusion can be found in Fig. 1. These patients were then matched to patients who underwent an isolated MPFLR based on demographics (age, gender, and BMI) and follow-up time. There were no significant differences in demographic variables between the groups. A comparison of patient demographics can be further illustrated in Table 1. 


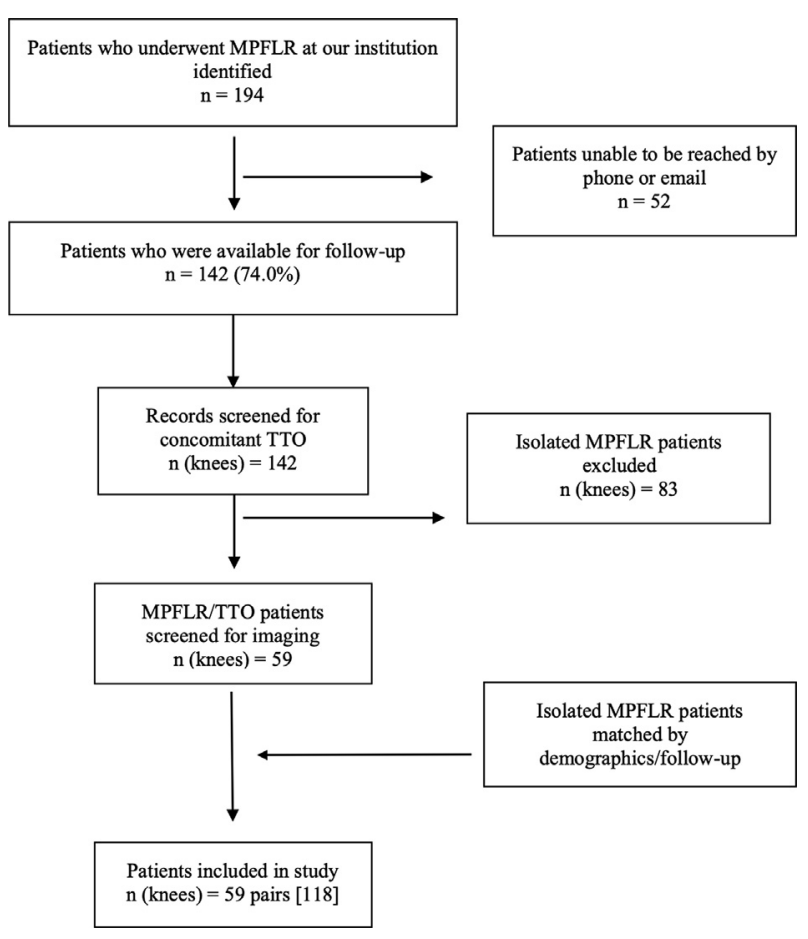

Fig. 1. Flow diagram of total patient inclusion/exclusion.

Table 1

Demographics.

\begin{tabular}{lll}
\hline Characteristics & MPFL & MPFL/TTO \\
\hline $\mathrm{N}$ & 59 & 59 \\
Gender (female) & $45(76.3 \%)$ & $45(76.3 \%)$ \\
Mean age (years) & $25.0 \pm 9.0$ & $25.0 \pm 8.9$ \\
Follow-up (months) & $49.0 \pm 27.0$ & $49.0 \pm 27.9$ \\
TT-TG distance (mm) & $14.1 \pm 2.8$ & $19.8 \pm 3.9$ \\
\hline
\end{tabular}

MPFL, medial patellofemoral ligament; TTO, tibial tubercle osteotomy; TT-TG, tibial tuberosity trochlear groove.

\section{Patient-reported outcomes}

At final follow-up, there were no significant differences in pain scores at rest or with activity, measured by both VAS and Kujala. There was no significant difference in patient satisfaction or the percentage of patients in either group that would repeat the procedure if given the choice. A comparison of patient-reported outcomes can be further illustrated in Table 2.

\section{Complications and revision surgeries}

One patient in each group (1.7\%) experienced recurrent instability postoperatively. Five patients in each group $(8.5 \%)$ went on to require another surgery. Revision surgeries included manipulation under anaesthesia (MUA), hardware removal, irrigation, and debridement, a

Table 2

Patient-reported outcomes.

\begin{tabular}{llll}
\hline Outcome measure & MPFLR & MPFLR/TTO & $p$ value \\
\hline $\mathrm{N}$ & 59 & 59 & - \\
VAS (at rest) & $1.48 \pm 2.0$ & $1.49 \pm 2.1$ & 0.972 \\
VAS (with activity) & $2.84 \pm 2.7$ & $2.66 \pm 2.7$ & 0.712 \\
Kujala & $86.3 \pm 13.3$ & $83.9 \pm 16.9$ & 0.375 \\
Satisfaction (\%) & $86.1 \% \pm 24.2 \%$ & $81.2 \% \pm 27.9 \%$ & 0.311 \\
Would repeat (\%) & $78.0 \% \pm 41.8 \%$ & $86.0 \% \pm 34.5 \%$ & 0.259 \\
\hline
\end{tabular}

MPFLR, medial patellofemoral ligament reconstruction; TTO, tibial tubercle osteotomy; VAS, visual analogue scale.
Table 3

Complications and further procedures.

\begin{tabular}{llll}
\hline Complication & MPFLR & MPFLR/TTO & $p$ value \\
\hline $\mathbf{N}$ & 59 & 59 & - \\
Recurrent instability & $1(1.7 \%)$ & $1(1.7 \%)$ & $>0.99$ \\
Further procedures & $5(8.5 \%)$ & $5(8.5 \%)$ & $>0.99$ \\
\hline MUA & 4 & 0 & 0.12 \\
Removal of hardware & 0 & 3 & 0.24 \\
I\&D & 0 & 1 & $>0.99$ \\
TKA & 0 & 1 & $>0.99$ \\
TTO & 1 & 0 & $>0.99$ \\
\hline
\end{tabular}

MPFLR, medial patellofemoral ligament reconstruction; MUA, manipulation under anaesthesia; I\&D, irrigation and debridement; TKA, total knee arthroplasty; TTO, tibial tubercle osteotomy.

subsequent TTO in an isolated MPFL patient and a total knee arthroplasty. Further detail can be seen in Table 3.

\section{Discussion}

The most important finding in this study was that at a mean of 4 years postoperatively, patients who underwent MPFLR with concomitant TTO did not differ in pain scores, satisfaction, or number of revision surgeries when compared with those treated with an isolated MPFLR. The overall functional outcome score was high, and low pain scores were reported. In addition, the vast majority of the patients were satisfied with long-term outcomes and would repeat the surgery if given the choice. Despite concerns that the bony realignment and additional hardware may cause increased pain, this does not appear to be the case. The primary purpose of this study was to assess the impact of a concomitant TTO on patientreported outcomes compared with an isolated MPFL reconstruction in the management of patellar instability. Although the matched patient cohorts demonstrated no difference in recurrence, this finding was not a goal of the present study.

Although evidence exists supporting the roles trochlear morphology and bony malalignment play in patellar instability, concerns regarding the complications introduced with bony realignment procedures remain, leading some orthopaedic surgeons to opt out of the addition of a TTO even if indicated based on imaging parameters [15]. A recent study by Erickson et al. [10] demonstrated positive outcomes after isolated MPFLR irrespective of mean trochlear height, TT-TG distance, and patellar height, positing that the addition of a TTO may not be necessary. Of note, this study only had one year of follow-up in $80 \%$ of their cohort, and almost half of the cohort was lost to follow-up by year 2 . There is therefore a high risk of bias in this study, as it is unknown how a large portion of their patient cohort is doing clinically, and the results are limited to a very short follow-up period.

The present study demonstrates that with longer-term follow-up, a bony procedure when clinically indicated is a safe and efficacious addition to standard MPFLR. However, the similar outcomes reported between groups does not indicate that the addition of a TTO is expendable. The patients undergoing isolated MPFLR and TTO/MPFLR would ideally be matched by anatomic risk factors including TT-TG distance, as it has been demonstrated that patients with larger TT-TG distance are intrinsically more likely to re-dislocate and thus would be expected to have higher rates of recurrence postoperatively [17]. Surgeons at our institution consequently recommend including a TTO for any patient with a TT-TG distance $>18 \mathrm{~mm}$. The TT-TG distances are thus inherently different between the two treatment groups, and the overall risk of recurrence cannot be considered equal.

The results of this study corroborate recent literature that has demonstrated that the addition of TTO to MPFLR results in the same good functional outcomes, patient-reported pain score, and low rate of recurrence at long-term follow-up [12,18]. Neri et al. [12] looked at 133 MPFLRs with an average of 6-year follow-up (minimum of 4 years) and found no difference in IKDC or Kujala functional scores among patients 
who underwent isolated MPFLR compared with MPFLR with TTO. Mulliez et al. [18] prospectively analysed 129 MPFLRs where approximately one-third of cases were supplemented with a TTO and found that there was no difference between groups clinically or radiographically at 2 years of follow-up. Of note however is that all patients included in both of the aforementioned studies underwent MPFLR with autograft, as opposed to the present study in which all patients underwent MPFLR with allograft.

The present study demonstrated no difference in pain or function based on VAS and Kujala respectively at a mean of 4 years of followup. There was also no difference in patient satisfaction or the proportion of patients that would undergo the same operation again if given the choice. Although not statistically significant, it was interesting to note that despite an equal number of additional required surgeries, MUA was exclusively seen in the isolated MPFL cohort, whereas hardware removal was exclusively seen in the MPFL/TTO cohort at the tibial osteotomy site. It would be interesting to explore further in a larger patient sample why stiffness seemed to be a more prevalent problem among isolated MPFLR compared with MPFLR with concomitant TTO.

\section{Limitations}

There were some limitations to this study. First, this is a retrospective analysis, which introduces an element of selection bias. However, this was a multisurgeon study, which may make this cohort more generalisable to the population. We also tried to limit bias with over $70 \%$ follow-up on all patients who underwent MPFLR. Although it would be interesting to compare our results to patients undergoing autograft, in our clinical practice, we predominantly use allograft, and thus, this is not feasible. Furthermore, the lack of preoperative scores is a limitation. Finally, at our institution, surgeons standardly perform TTO on all patients indicated based on TT-TG distance $>18 \mathrm{~mm}$. Therefore, there is a natural difference present between the groups as the average TT-TG is higher in the MPFL/TTO group than in the MPFL group.

\section{Conclusion}

Matched patients undergoing MPFLR with TTO compared with isolated MPFLR demonstrate no statistically significant difference in patient-reported outcomes, levels of pain, and satisfaction postoperatively. Furthermore, the addition of a TTO does not increase the risk of further surgery or complications.

\section{Declaration of competing interest}

There are no conflicts of interest to report.

\section{Appendix A. Supplementary data}

Supplementary data to this article can be found online at https ://doi.org/10.1016/j.jisako.2021.10.004.

\section{References}

[1] Stefancin JJ, Parker RD. First-time traumatic patellar dislocation: a systematic review. Clin Orthop Relat Res 2007;455:93-101.

[2] Sanders TL, Pareek A, Hewett TE, et al. Incidence of first-time lateral patellar dislocation: a 21-year population-based study. Sport Health 2018;10(2):146-51.

[3] Atkin DM, Fithian DC, Marangi KS, et al. Characteristics of patients with primary acute lateral patellar dislocation and their recovery within the first 6 months of injury. Am J Sports Med 2000;28(4):472-9.

[4] Hsiao M, Owens BD, Burks R, et al. Incidence of acute traumatic patellar dislocation among active-duty United States military service members. Am J Sports Med 2010; 38(10):1997-2004.

[5] Fithian DC, Paxton EW, Stone ML, et al. Epidemiology and natural history of acute patellar dislocation. Am J Sports Med 2004;32(5):1114-21.

[6] Sappey-Marinier E, Sonnery-Cottet B, O'Loughlin P, et al. Clinical outcomes and predictive factors for failure with isolated MPFL reconstruction for recurrent patellar instability: a series of 211 reconstructions with a minimum follow-up of 3 years. Am J Sports Med 2019;47(6):1323-30.

[7] Liu JN, Brady JM, Kalbian IL, et al. Clinical outcomes after isolated medial patellofemoral ligament reconstruction for patellar instability among patients with trochlear dysplasia. Am J Sports Med 2018;46(4):883-9.

[8] Shamrock AG, Day MA, Duchman KR, et al. Medial patellofemoral ligament reconstruction in skeletally immature patients: a systematic review and metaanalysis. Orthop J Sports Med 2019;7(7). 2325967119855023.

[9] Schneider DK, Grawe B, Magnussen RA, et al. Outcomes after isolated medial patellofemoral ligament reconstruction for the treatment of recurrent lateral patellar dislocations: a systematic review and meta-analysis. Am J Sports Med 2016; 44(11):2993-3005.

[10] Erickson BJ, Nguyen J, Gasik K, et al. Isolated medial patellofemoral ligament reconstruction for patellar instability regardless of tibial tubercle-trochlear groove distance and patellar height: outcomes at 1 and 2 years. Am J Sports Med 2019; 47(6):1331-7.

[11] Liu JN, Steinhaus ME, Kalbian IL, et al. Patellar instability management: a survey of the International Patellofemoral Study Group. Am J Sports Med 2018;46(13):3299-306.

[12] Neri T, Parker DA, Beach A, et al. Medial patellofemoral ligament reconstruction with or without tibial tubercle transfer is an effective treatment for patellofemoral instability. Knee Surg Sports Traumatol Arthrosc 2019;27(3):805-13.

[13] McNeilan RJ, Everhart JS, Mescher PK, et al. Graft choice in isolated medial patellofemoral ligament reconstruction: a systematic review with meta-analysis of rates of recurrent instability and patient-reported outcomes for autograft, allograft, and synthetic options. Arthroscopy 2018;34(4):1340-54.

[14] Desai VS, Tagliero AJ, Parkes CW, et al. Systematic review of medial patellofemoral ligament reconstruction techniques: comparison of patellar bone socket and cortical surface fixation techniques. Arthroscopy 2019;35(5):1618-28.

[15] Post WR, Fithian DC. Patellofemoral instability: a consensus statement from the AOSSM/PFF patellofemoral instability workshop. Orthop J Sports Med 2018;6(1). 2325967117750352.

[16] Stupay KL, Swart E, Shubin Stein BE. Widespread implementation of medial patellofemoral ligament reconstruction for recurrent patellar instability maintains functional outcomes at midterm to long-term follow-up while decreasing complication rates: a systematic review. Arthroscopy 2015;31(7):1372-80.

[17] Cooney AD, Kazi Z, Caplan N, et al. The relationship between quadriceps angle and tibial tuberosity-trochlear groove distance in patients with patellar instability. Knee Surg Sports Traumatol Arthrosc 2012;20(12):2399-404.

[18] Mulliez A, Lambrecht D, Verbruggen D, et al. Clinical outcome in MPFL reconstruction with and without tuberositas transposition. Knee Surg Sports Traumatol Arthrosc 2017;25(9):2708-14. 